\title{
Hypertrophic granulomatous cranial pachymeningitis causing progressive blindness in a chronic dialysis patient
}

\author{
EARL R. FERINGA ${ }^{1}$ AND LEE WEATHERBEE \\ From the Departments of Neurology and Pathology, Veterans Administration Hospital, Ann Arbor, Michigan, \\ U.S.A.
}

SYNOPSIS A patient on chronic haemodialysis because of renal failure developed progressive visual field loss and eventual blindness. A postmortem examination indicated that the blindness was caused by granulomatous thickening of the pachymeninges which constricted the optic nerves as they passed through the optic foramina. Exhaustive bacteriological and histopathological studies failed to identify a specific cause for this hypertrophic cranial pachymeningitis.

A variety of unique disease processes in patients with chronic renal failure whose lives have been prolonged by haemodialysis have been described in recent years. In most cases, it is unclear whether these unusual pathological states are coincidentally associated with chronic renal disease and haemodialysis, are caused by supraend-stage renal disease, or are the result of haemodialysis. In this paper we report a patient who developed progressive visual field loss and eventually became blind. He had been on haemodialysis for two years because of chronic renal failure due to rapidly progressive glomerulonephritis. At postmortem examination both optic nerves were compressed by hypertrophic changes in the dura mater. The cause of these changes is unknown.

\section{CASE HISTORY}

In October 1971, a 50 year old white man first noted malaise, weakness, nausea, and anorexia. He had peripheral oedema, an elevated serum potassium $(8.1 \mathrm{mmol} / \mathrm{l})$, a blood urea nitrogen of $80.3 \mathrm{mmol} / \mathrm{l}$, and a creatinine of $2376.0 \mu \mathrm{mol} / \mathrm{l}(27 \mathrm{mg} / \mathrm{dl})$. The cause of his renal disease was not clearly defined. Antinuclear antibody tests, rheumatoid factor studies, and antistreptolysin 0 titres were all normal.

\footnotetext{
1 Reprint requests: Dr E. R. Feringa, Chief, Neurology Service Veterans Administration Hospital, 2215 Fuller Rd., Ann Arbor, Michigan 48105, U.S.A.

(Accepted 24 June 1975.)
}

He was first treated with peritoneal dialysis; then an arteriovenous fistula was placed and he was started on a programme of haemodialysis.

Haemodialysis was continued three times a week with apparently good control of his uraemia. In March 1973 he noted the onset of hoarseness, a headache on the left side, a pressure sensation in his left ear, and a decrease in hearing acuity. Direct laryngoscopy demonstrated paralysis of the left vocal cord. Five days later another examiner felt that the vocal cords were normal, although the patient continued to have hoarseness. In May 1973 he complained of visual difficulty and was found to have a visual acuity (corrected) of 20/25 in the right eye and 20/60-1 in the left. Near vision was Jaeger 0 in the right eye and Jaeger 13 in the left. Visual field examination demonstrated a superior lateral visual field defect in the left eye and the suggestion of a similar defect in the right eye.

A complete neurological evaluation was done in June 1973. His visual fields appeared to be full on confrontation testing. The disc margin was indistinct on the nasal side in the left optic fundus, but was not considered definitely abnormal. The left palate was weak. There was decreased bulk and power of the left sternocleidomastoid muscle and of the upper fibres of the left trapezius muscle. The tongue appeared to deviate slightly to the left when protruded. Except for minimal evidence of a peripheral neuropathy manifest by decreased sensation and decreased deep tendon reflexes distally and symmetrically, the findings of the rest of the neurological 


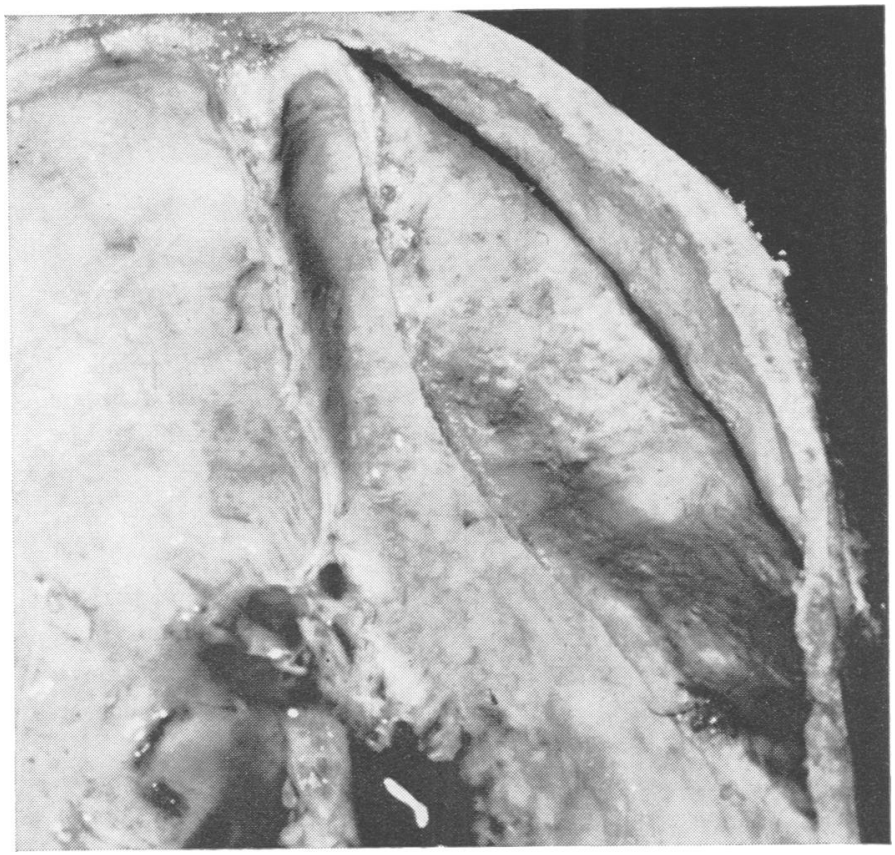

FIG. 1 A gross view of the skull cap at necropsy showing the thickened dura mater. Note the complete occlusion of the superior sagittal sinus.

examination were normal. A chest radiograph at this time revealed two small 'coin lesions' in the right inferior lung field. These were not present on earlier films. An electroencephalogram showed excessive anterior amplitude with bianterior slow activity and bitemporal slow activity but no focal findings. A lumbar puncture in June 1973 showed an opening pressure of 220 to $230 \mathrm{~mm}$ of spinal fluid. The fluid contained three red cells per $\mathrm{ml}$ and no white cells. The protein was $0.18 \mathrm{~g} / \mathrm{l}$, the glucose $3.55 \mathrm{mmol} / \mathrm{l}$ with a simultaneous blood glucose of $5.72 \mathrm{mmol} / \mathrm{l}$. A Gram stained smear of the spinal fluid showed no bacteria. Fungus cultures, bacterial cultures for pyogens and for tubercle bacilli, an India ink preparation, and the VDRL test were all negative.

An extensive evaluation for possible fungal or neoplastic disease was begun. Serum for fungal antigens was negative for Blastomyces dermatitidis, Candida albicans, Coccidioides immitis, and Histoplasma capsulatum (three strains). The sputum was examined for malignant cells on three occasions and none was found. Bronchial washings and bronchial brushings from three separate areas were obtained and all were negative for neoplasia. An upper gastrointestinal radiographic series was interpreted as showing an antral mass lesion with an ulcerated crater and two duodenal diverticulae. Gastric washings and gastric brushings showed no evidence of neoplasia.
Fibreoptic gastroscopy and biopsy failed to demonstrate tumour. A barium enema showed diverticulosis without diverticulitis.

Skull radiographs with particular views of the jugular foramina and the optic foramina were normal. A brain isotope scan was read as probably abnormal with increased uptake bilaterally in the frontal region and near the sella turcica, but no definite mass could be identified. Periodic repeat chest radiographs demonstrated continued gradual enlargement of the 'coin lesions' in the right lower lung zone.

A sigmoidoscopy and a liver scan were normal. A slide test for antinucleoproteins associated with lupus erythematosus was negative. The antinuclear antibody test was negative again. A serum fluorescent treponema antibody test was negative. The sedimentation rate of the blood was $64 \mathrm{~mm} / \mathrm{h}$. A tuberculosis skin test with purified protein derivative was negative at 48 hours on two occasions.

In June 1973, a re-examination showed the corrected visual acuity in the right eye to be $20 / 30+2$, and in the left eye, 20/300. The visual fields were constricted, and examination was limited by the patient's poor cooperation, but it was felt that there was a bitemporal superior field defect which was worse in the left eye than in the right. Neurological examination showed a questionable left superior quadrant defect on confrontation visual fields. 


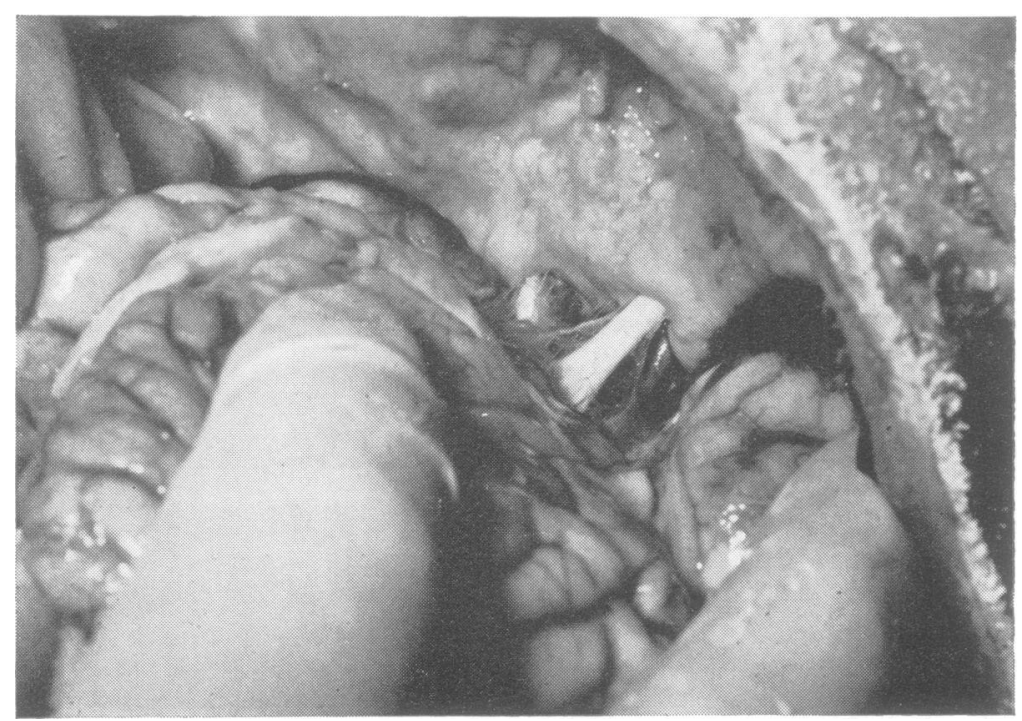

FIG. 2 The optic nerves were compressed by the greatly thickened dura mater. Dura mater at the base of the skull was 2 to $3 \mathrm{~mm}$ thick and had a lumpy appearance and a yellowish colour in the fresh state.

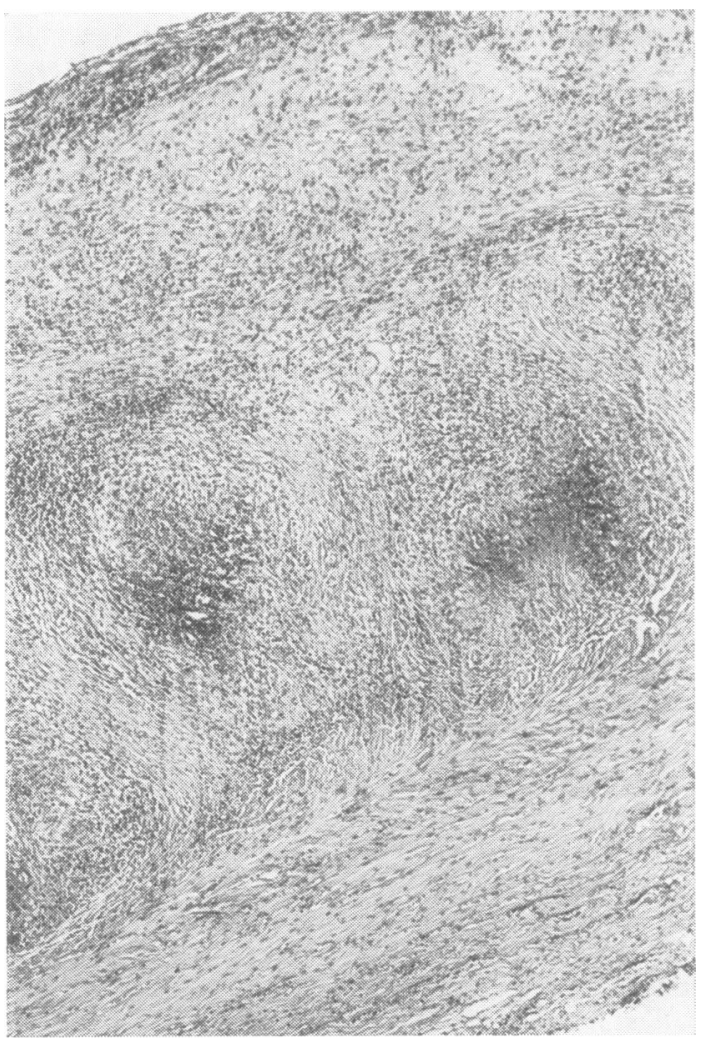

FIG. 3 Area of the thickened dura mater showing areas of focal granulomatous inflammation and necrosis. Note the increased collagen and foci of chronic inflammatory cellular infiltrates. $\times 50$.
The patient complained of pain in the left jaw and of left ear fullness. His tonsils were enlarged and had pus-filled crypts. There were decreased bulk and power of the sternocleidomastoid and the trapezius muscles on the left. A subsequent EMG, however, failed to reveal any significant evidence of denervation. A second set of skull films was reviewed with the neuroradiologist, with special attention given to base views, but no abnormality was found. The patient insisted on being discharged from the hospital and was allowed to go home with the understanding that further evaluation of his lung nodules and neurological status was imperative just as soon as he would be willing to return to the hospital.

Ten days later, while the patient was at home, he lost all vision. He refused hospitalization, but for the next week he claimed that after dialysis he sometimes could see for a few minutes; then he would become blind again. When he was admitted one week after the onset of blindness, his pupils were found to be dilated with no reaction to light. Definite optic atrophy was not identified. There was no perception of light.

A repeat gastroscopy demonstrated chronic inflammation of the stomach lining with no evidence of tumour. A needle biopsy of the lung revealed acute and chronic inflammatory changes but no evidence of tumour. A repeat neurological examination three weeks after the onset of blindness revealed a normal mental status, except that the patient was very anxious about his condition. No light perception was possible with either eye. The right pupil was slightly larger and more irregular than the left, but both pupils were dilated widely. The right pupil 
reacted slightly to direct light stimulation, but there was no consensual response. The left pupil did not respond to any light stimulus. The patient's voice remained hoarse. There was minimal decrease in the nasolabial fold on the right as compared with the left.

Another lumbar puncture revealed normal pressure and crystal clear fluid. Four red cells per $\mathrm{mm}^{3}$ and no white cells were seen on microscopic examination. The spinal fluid protein was $0.15 \mathrm{~g} / \mathrm{l}$ and the glucose $3.94 \mathrm{mmol} / \mathrm{l}$. The VDRL was negative. India ink preparations, cultures for fungus, and smears and cultures for bacteria were negative. Four vessel cerebral angiography was done and no definite abnormality was found. The patient was discharged without definite diagnosis and continued on his haemodialysis programme.

In October the patient had a regularly scheduled haemodialysis. After returning home he developed an extremely severe headache. He became less responsive and lapsed into coma within three hours. A lumbar puncture revealed an opening pressure of $500 \mathrm{~mm}$ of grossly bloody spinal fluid. When the fluid was centrifuged, the supernatant was mildly xanthochromic. Despite treatment, he died, two years after the onset of haemodialysis for renal disease and three months after the onset of blindness.

\section{PATHOLOGICAL FINDINGS}

A large recent intracerebral haematoma involved the surface and extended deeply into the occipital and parietal areas of the right cerebral hemisphere. The leptomeninges were otherwise smooth and glistening.

The dura mater was markedly thickened, irregular, and yellow coloured. Over the base of the skull the dura mater was 2 to $3 \mathrm{~mm}$ thick. Over the convexities it was 1 to $2 \mathrm{~mm}$ thick (Fig. 1). The dura mater was particularly thick in the area of the optic foramina and appeared to be constricting the optic nerves bilaterally (Fig. 2). Thickened and irregular dura mater was noted also in the area of the jugular foramen bilaterally, and there was destruction of bone in that region. In all areas the microscopic changes of the dura mater were rather uniform and consisted of confluent granulomas, often with prominent areas of necrosis and considerable fibrosis (Figs 3 and 4). Infiltrates of chronic inflammatory cells included lymphocytes, plasma cells, and scattered eosinophils. A few giant cells were seen adjacent to the necrosis.

There was erosion of the petrous portion of the left temporal bone which permitted communication of the left mastoid air cells with the epidural space. No definite aetiology for the destruction of the bone was determined. The superior sagittal sinus was obliterated by granulomatous pachymeningitis.

A broad based $3 \times 1.5 \mathrm{~cm}$ anterior lower lobe scar was seen on the pleural surface of the right lung. Beneath this pleural scar there was a cheesy necrotic area measuring $5 \times 4 \mathrm{~cm}$. Microscopically, this lesion consisted of granulomatous tissue with lymphocytes and eosinophils similar to the granulomatous involvement of the dura mater. The necrosis, however, was more extensive and acute inflammatory cells were more prominent.

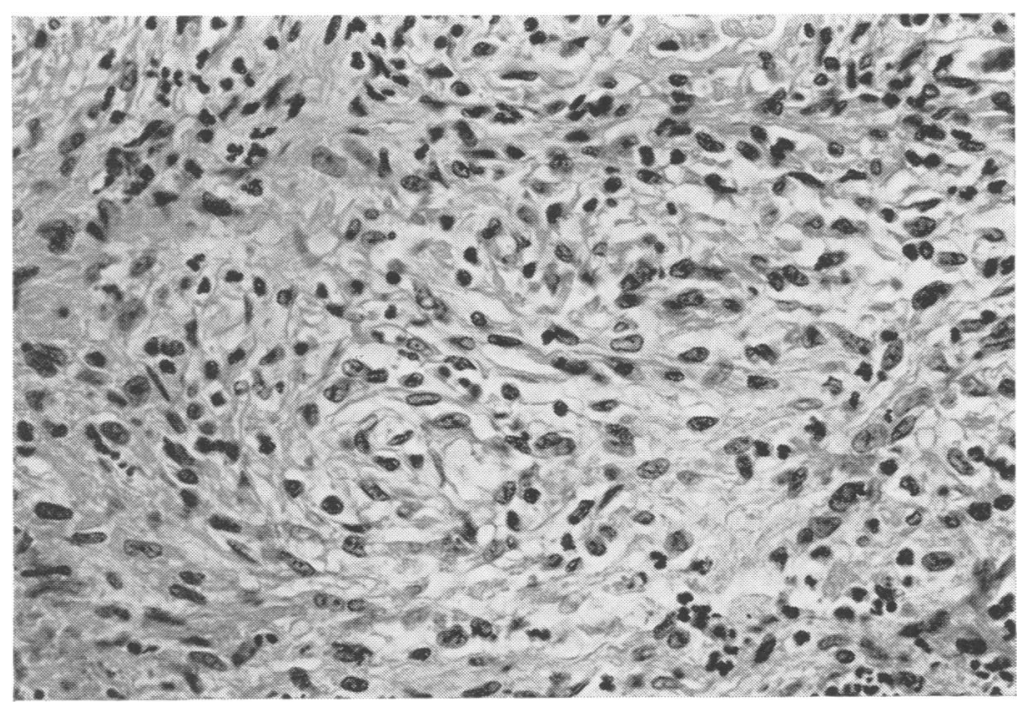

FIG. 4 High power view of an area showing histiocytes characteristic of granulomatous inflammation. Scattered lymphocytes are also seen and occasional polymorphonuclear leucocytes are seen at the edges of the picture adjacent to areas of necrosis. $\times 440$. 
The kidneys weighed $75 \mathrm{~g}$ each. The capsules stripped with ease but the cortical surface was finely granular and pale tan-white. The cortex averaged $2 \mathrm{~mm}$ in thickness. The cortical medullary junctions were indistinct and the medulla was poorly striated. The pyramids were blunted and the pelves and calyces slightly dilated. There was increased peripelvic fat and the renal vessels were patent. Nearly all glomeruli were sclerotic. Arterial and arteriolosclerosis was prominent. Focal chronic inflammatory infiltrates, tubular atrophy and dilatation, and very rare foci of acute inflammatory cells were noted adjacent to small areas of necrosis.

No abnormalities were noted in the stomach.

The granulomatous areas of the dura mater and lung were examined further by special stains. Silver methenamine and PAS stains for fungi were negative. Levaditi stains for spirochaetes and acid fast stains for tubercle bacilli were also negative. Brown and Brenn stains did not demonstrate the presence of bacteria. Cultures of the dura mater for mycobacteria, fungi, and pyogenic bacteria were negative.

\section{DISCUSSION}

The existence of disease states which cause hypertrophy of the pachymeninges and neurological abnormalities have been discussed by several authors. Gowers (1893) describes pachymeningitis as either external or internal depending on the direction of insult to the dura mater. In his description 'external pachymeningitis is almost always secondary to injury or to adjacent disease...' He describes cases in which local trauma or infection of the bones of the skull resulted in local inflammation of and some thickening of the dura mater. He does not discuss a more diffuse problem such as is demonstrated by our patient. While our patient did have ear symptoms of fullness and hearing loss and at postmortem examination showed local destruction of bone in the area of the left petrous pyramid, the involvement of the dura mater was not local but rather spread extensively over the entire inner table of the skull.

Gowers further divides internal pachymeningitis into purulent and haemorrhagic types. The purulent type seems to be associated with purulent infection of the subarachnoid space, an infection which our patient clearly did not have. His 'haemorrhagic internal pachymeningitis' seems to correlate best with what would now be called chronic subdural haematoma. The patho- logical findings in his cases of much blood and blood pigment and many large capillaries in the hypertrophic dura mater are all very different from the granulomatous tissue in our case.

Patients on chronic haemodialysis have a tendency to bleed for several reasons. In our patient the terminal event was a massive intracerebral haematoma. However, the clinical course of this patient was not one of multiple clinical episodes compatible with repeated subdural haemorrhages. When checked at various times the spinal fluid never showed more than a few red blood cells and always had low normal CSF proteins. The findings at postmortem examination showed only minimal evidence of haemorrhage. This haemorrhage was very acute. It involved only the inner surface of the dura mater adjacent to the area of acute intracerebral haemorrhage which was the patient's terminal event. The layering of fibrous tissue, excessive blood pigments, and multiple large capillary spaces described by Gowers were all missing in this case.

While CSF findings are not described by Gowers, his description of the involvement of adjacent arachnoid and pia mater by '... peculiar membranous tissue' would make it unlikely that such patients would have normal CSF. While Gowers's list of symptoms ascribed to haemorrhagic internal pachymeningitis resembles a list of the ills which befall mankind's brain, blindness is notably absent.

Wilson (1940) has also discussed pachymeningitis. His discussion parallels that of Gowers. Again, the external variety of pachymeningitis is most closely associated with local disease processes and is not described as causing a diffuse cranial hypertrophic reaction. His description of 'pachymeningitis haemorrhagica interna' again seems to correlate poorly with the history and findings in our case, and much better with chronic subdural haematoma. Blindness is not described as a sign of this disease by Wilson.

In both Gowers's and Wilson's discussions the aetiology of many cases is ascribed to syphilis, tuberculosis, or perhaps alcohol. Modern neuropathologists would probably hold that these discussions present inadequate proof to establish a cause and effect relationship, except in the case where the infection of the dura mater was associated with leptomeningitis caused by syphilis or tuberculosis. The more chronic cases without 
involvement of the leptomeninges are probably simply unexplained.

Better known is hypertrophic cervical pachymeningitis (Charcot, 1873). This disease is not limited to the cervical spinal area, nor is it limited to the dura mater. Rather 'the neighbouring leptomeninx always suffers as well, becoming opaque and thick, and firmly united to the dura and cord ...' (Wilson, 1940). But this disease is not described as involving the intracranial dura mater, and blindness is not a result of this infection. Hypertrophic pachymeningitis affecting areas other than the spinal dura mater is not described in modern neurology texts.

Åström and Lidholm (1963) described a case of granulomatous inflammation of the orbit which, at postmortem examination, was found to have spread into the cerebral subarachnoid space as well as to other adjacent tissues. This occurred in a patient with well-established periarteritis nodosa. The authors discuss a possible relation to Wegener's granulomatosis. The lesions described are very different from those of our case. (1) Grossly, the lesions involved the subarachnoid space, while our case had a normal subarachnoid area. (2) Histologically, the lesions were characterized by vasculitis with associated chronic and acute infection, while our case showed almost no vascular involvement. (3) Clinically, the intracranial lesion was an incidental terminal spread of an otherwise obvious orbital granuloma, while in our case the presence of granuloma intracranially was completely hidden until postmortem examination.

We have also considered that our patient's disease process might be an example of Wegner's granulomatosis. If his kidney disease be considered along with his lung lesions and cerebral symptoms, this might be a unifying diagnosis. In the end, however, we have rejected this diagnosis on both clinical and pathological grounds.

Clinically, Wegener's granulomatosis is at best a poorly defined entity (Wolff et al., 1974). Almost all patients with this disease show evidence of sinus or nasopharyngeal inflammatory disease. Symptoms of such were lacking in this patient, and even a retrospective review of two sets of sinus films taken after his symptoms began revealed only minimal thickening of the mucosa of the ethmoid sinuses. No active sinus inflammation or infection can be identified on any film.
A variety of neurological complaints have been listed as associated with Wegener's granulomatosis (Drachman, 1963). Patients have lesions caused by vasculitis. Peripheral neuropathies are the most common neurological manifestation. Ocular signs associated with necrotic granulomas of the orbit, of the eye, and lesions of the third, fourth, and sixth cranial nerves are described (Straatsma, 1957), but visual field loss or blindness is notably absent. Although survival up to three years has been reported in patients with Wegener's granulomatosis (Godman and Churg, 1954), most cases die in two to five months. If the original kidney disease be considered part of the same disease, our patient had a survival of two years.

One report (Rogers and Roberto, 1956) describes focal involvement of the dura mater adjacent to the left superior orbital fissure with complete internal and external ophthalmoplegia on that side. An exploratory subtemporal craniectomy revealed a markedly thickened dura mater with yellowish-grey granulations, an increased vascularity, and a gelatinous appearance. Postoperatively, the patient became blind in that eye. That patient died five months later after he developed both extensive local disease in the eye and orbit and extensive upper and lower respiratory disease. The necropsy showed '... marked fibrosis and inflammation of the dura due to acute vasculitis'. The extent of involvement of the dura mater, the leptomeninges, and/or the brain is not described, nor is blindness on the right side.

Pathologically, Wegener's granulomatosis is a necrotizing granulomatous vasculitis (Godman and Churg, 1954). In our patient, some blood vessels were involved in the granulomatous processes, but this appeared to be quite coincidental to the diffuse involvement of adjacent tissue. No evidence of primary granulomatous vasculitis was found in evaluation of dura mater, kidney, or lung lesions.

We report a case of progressive blindness in a patient on a programme of chronic renal dialysis. At postmortem examination a chronic cerebral granulomatous pachymeningitis of uncertain aetiology was found to have constricted both optic nerves. Exhaustive efforts to identify the cause of this granulomatous thickening of the dura mater have been unsuccessful. Because 
of the palisading histiocytes, the character of the necrosis, and the scattered eosinophils, we have concluded that the best pathological description is that of nonspecific allergic granulomatosis. We can find no reports of similar cases. We hope our report will stimulate others to be aware of this unusual cause of progressive visual field loss and eventual blindness.

This project was supported by institutional research funds of the Veterans Administration Hospital at Ann Arbor, Michigan.

\section{REFERENCES}

Åström, K. E., and Lidholm, S. O. (1963). Extensive intracranial lesions in a case of orbital non-specific granuloma combined with polyarteritis nodosa. Journal of Clinical Pathology, 16, 137-143.
Charcot, J. M. (1873). Séance du 13 mai. Comptes Rendus des Séances de la Société de Biologie, Serie 5e iii, 1871, 32-38.

Drachman, D. A. (1963). Neurological complications of Wegener's granulomatosis. Archives of Neurology, 8, 145-155.

Godman, G., and Churg, J. (1954). Wegener's granulomatosis. Archives of Pathology, 58, 533-553.

Gowers, W. R. (1893). A Manual of Diseases of the Nervous System, vol. 2, 2nd edn., pp. 323-328. Hafner: Darien.

Rogers, J. V., and Roberto, A. E. (1956). Circumscribed pulmonary lesions in periarteritis nodosa and Wegener's granulomatosis. American Journal of Roentgenology, 76, 88-93.

Straatsma, B. R. (1957). Ocular manifestations of Wegener's granulomatosis. American Journal of Ophthalmology, 44, 789-799.

Wilson, S. A. K. (1940). Pachymeningitis. In Neurology, vol. I, pp. 4-8. Edited by A. N. Bruce. Butterworths: London.

Wolff, S. M., Fauci, A. S., Horn, R. G., and Dale, D. C. (1974). Wegener's granulomatosis. Annals of Internal Medicine, 81, 513-525. 